\title{
Correction to: Catalytic Decomposition of Hydrogen-lodide Over Nanocrystalline Ceria Promoted by Transition Metal Oxides for Hydrogen Production in Sulfur-lodine Thermo-Chemical Cycle
}

\author{
Amit Singhania ${ }^{1} \cdot$ Ashok N. Bhaskarwar $^{1} \cdot$ Parvatalu Damaraju $^{2} \cdot$ Satinath Banerjee $^{2} \cdot$ Bharat Bhargava $^{2}$
}

Published online: 23 March 2018

(c) Springer Science+Business Media, LLC, part of Springer Nature 2018

\section{Correction to: Catalysis Letters \\ https://doi.org/10.1007/s10562-017-2240-0}

In the original version of this article, four co-authors and some texts of acknowledgement were missing.

This has been corrected with this erratum.
Acknowledgements Financial assistance from ONGC Centre for the work and fellowship to one of the authors "Amit Singhania" is gratefully acknowledged.

The original article can be found online at https://doi.org/10.1007/ s10562-017-2240-0.

Amit Singhania amit.singhania2008@gmail.com

Ashok N. Bhaskarwar

ashoknbhaskarwar@yahoo.co.in

Parvatalu Damaraju

parvatalu_d@ongc.co.in; parvatalu@gmail.com

Satinath Banerjee

satinath_banerjee@ongc.co.in; banerjeesbi@gmail.com

Bharat Bhargava

bhargavabh@ongc.co.in; bbhargava51@gmail.com

1 Department of Chemical Engineering, Indian Institute of Technology, Delhi, Hauz Khas, New Delhi 110016, India

2 ONGC Energy Centre, 2nd Floor, Core-2, Scope Minar, Laxmi Nagar, New Delhi 110092, India 